\title{
“Unconventionally Shaped” Antenna Design for UHF RFID Tags
}

\author{
Silong Wang $(\mathbb{D}$, Yulong Liu, and Terry Tao Ye $\mathbb{D}$ \\ Department of Electrical and Electronic Engineering and University Key Laboratory of Advanced Wireless Communications of \\ Guangdong Province, Southern University of Science and Technology, Shenzhen, China
}

Correspondence should be addressed to Terry Tao Ye; yet@sustech.edu.cn

Received 11 March 2021; Revised 31 May 2021; Accepted 11 June 2021; Published 1 July 2021

Academic Editor: Francesco D’Agostino

Copyright (c) 2021 Silong Wang et al. This is an open access article distributed under the Creative Commons Attribution License, which permits unrestricted use, distribution, and reproduction in any medium, provided the original work is properly cited.

UHF RFID tags need to be attached or embedded into various objects. Unlike traditional free-standing antennas, UHF antenna shapes and form factors may vary significantly. There have been no systematic methods that facilitate the design practice of antenna with unconventional shapes. In this paper, using the geometries of 26 English letters (in capital) as examples, we explore the general methodology of shape-specific antenna design. More specifically, we show that 26 letter geometries can be categorized into 9 groups, and the antennas in each group can be divided and conquered into standard baseline geometries. Through prototypes and measurements, we demonstrate that each letter-shaped antenna, although exhibiting different gains and radiations, can achieve satisfactory performance, as compared to standard UHF dipole antennas. Specifically, letters "M" and "J" achieve the longest reading range of more than 20 meters with a good radiation pattern, which is comparable or even better than many commercial UHF RFID tags.

\section{Introduction}

UHF radio frequency identification (RFID) systems have demonstrated great potentials in logistic, anticounterfeiting, and retail applications [1]. Unlike many other wireless transceiver devices that have free-standing antennas, RFID tags are required to be attached or embedded into various objects with different form factors. UHF antennas need to be compact, conformal, and conceivable, while still maintaining a satisfactory EM performance with low mass-production costs. The design of the tag antennas has always played a significant role in RFID implementation [2].

The most popular UHF RFID antenna structures are the meander-line dipole (MLD) and slotted-patch microstrip (SPM) antennas [3]. MLD and SPM geometries are relatively small in size, with satisfactory gain and low cost in mass production. T-match structure is commonly used to achieve impedance matching between the transponder chip and the MLD antennas [4]. Antenna's S11 parameters and other EM properties can be conveniently tuned by adjusting the positions and geometries of the T-match and antenna body.

RFID tags normally are packaged into labels, where the antennas are conformed to the rectangular form factor, of which MLD and SPM antenna geometries fit naturally [5].
However, as more and more RFID tags are no longer used as labels, antenna geometries are no longer constrained by conventional rectangular form factors.

The unconventional shape requirements of antenna may come from different scenarios. For example, RFID tags are embedded inside bottle caps as a measure of anticounterfeiting; antennas can be conformal to the shapes of component parts on the assembly line; and in many garment deployment applications, antennas can be embroidered on apparels [6], so antennas can be conceived as logos or decorative patterns. These scenarios all demand antennas with unconventional geometries.

Many unconventional antennas have been proposed in the past. Xi and Ye had proposed a tag antenna that can be mounted directly on the wine bottle neck [7]; the lettershaped antennas can be used as brand logos as well. Chung and Wong had proposed a Wang-shaped patch antenna conceived as a Chinese character; in the meantime, the antenna has a small aperture for wireless communication [8]. Mutlu et al. had proposed a chipless tag antenna based on the letter "ABC" [9]. Tedjini et al. had demonstrated an approach for designing an alphabet-shaped chipless RFID tag antenna with letters "a," "b," and "c" as templates; the letter geometries need to be placed with $15 \mathrm{~cm}$ separation. 
The chipless tag antenna can be identified through exploitation of resonant frequencies [10]. Keskilammi and Kivikoski had proposed a new design using text as a meander line to reduce the dimension of dipole antenna used in passive transponders [11]. The design proposed in the paper consisted of three phrases with 22 letters; half of the letters were used as a dipole arm, but the approach could not be generalized to other phrases. Singh et al. had presented a method to identify the letters with electromagnetic signature through RF signals [12]. This paper demonstrates that different letters exhibit different characteristics in frequency domain. Exploiting the form factor and geometries of letters and characters, other logo-based shapes have attracted many interests from antenna designers and package designers as well as fashion designers [13].

For MLD antennas, many standard design templates are available, which can greatly reduce the design cycle. However, for those unconventional antennas, the design practices are mostly based on try and error.

In this paper, we propose design procedures that can be used to design unconventionally shaped antennas. Particularly, we use the 26 English letters (in capital) as examples to demonstrate how to conform antenna geometries into particular shapes. Without loss of generality, we categorize the 26 letters into 9 groups, based on the letter's geometry of each group; we design one antenna as a template for each of the 9 groups. The bandwidth, S-parameters, and directivity will be comparable to those of standard MLD antennas of similar dimensions. Prototypes of each of the 9 templates are tested and prove that the design procedures are feasible and practical. Our proposed methodology can be extended to the design practice of other unconventionally shaped antennas.

With the increasing deployment of RFID tags, many RFID antennas are applied as logos or labels on objects, especially on garments and apparels. Letter shapes are the natural selection for logo geometries. Of course, there are countless other shapes that can be used as logos; the study in this work serves only as the initial steps for more unconventionally shaped antennas.

The paper is organized as follows. In Section 2, we analyze the T-match mechanism for impedance tuning in MLD antenna designs. In Section 3, we categorize the 26 English letters into 9 groups and present the steps in designing antennas of different shapes. Nine template antennas of each category are designed and prototyped; their testing results are analyzed in Section 4 . The design guidelines are summarized in Section 5.

\section{T-Match for Impedance Matching}

One of the objectives of designing an RFID antenna is to tune the impedance of the antenna to match with the chip to achieve maximum power transmission. The power transmission coefficient $\tau$ between the antenna and the chip can be expressed as [14]

$$
\tau=\frac{4 \operatorname{Re}\left\{Z_{a}\right\} \operatorname{Re}\left\{Z_{\text {chip }}\right\}}{\left|Z_{a}+Z_{\text {chip }}\right|^{2}},
$$

where $Z_{a}$ and $Z_{\text {chip }}$ are the impedance of the antenna and the chip, respectively. When $Z_{a}$ and $Z_{\text {chip }}$ are conjugated with each other, $\tau$ will reach its maximum value, that is, $\tau=1$, to achieve maximum power transmission efficiency.

As RFID chips are manufactured with standard IC foundry process, the chip's impedance is consistent and can be acquired through the manufacturer's datasheets. Impedance matching is achieved through the antenna design that uses the chip's parameters as reference (impedance, chip size, pad pitch, etc.). On most occasions, IC chip's impedance is capacitive at the operation frequency. Thus, an inductive matching structure needs to be introduced to antenna geometries.

T-match is a commonly used impedance matching technique in UHF RFID tag antenna design. The basic structure of the T-match is shown in Figure 1(a); it can be regarded as the combination of a dipole arm and an inductive loop.

Uda [15] had proposed an equivalent circuit of T-match as shown in Figure 1(b). The impedance at the feeding point of the T-match geometry can be given by

$$
Z_{a}=\frac{2 Z_{t}(1+\alpha)^{2} Z_{d}}{2 Z_{t}+(1+\alpha)^{2} Z_{d}}
$$

where $Z_{d}$ is the impedance of the dipole antenna without T-match structure and $Z_{t}$ is the input impedance of the transmission line that is decomposed from the inductive loop. As shown in Figure 1(b), the inductive loop is split into two parts; each part can be considered one transmission line with a length of $L / 2$ and is shorted at one end. Thus, $Z_{t}$ can be expressed as

$$
Z_{t}=j Z_{0} \tan \frac{k L}{2}
$$

where $k=2 \pi / \lambda$ and $Z_{0}$ is the characteristic impedance of transmission line and can be expressed as

$$
Z_{0}=276 \log _{10}\left(\frac{W}{\sqrt{2.0625 d_{1} d_{2}}}\right) \text {. }
$$

In addition, $\alpha=\left(\ln \left(W / 0.25 d_{1}\right) / \ln \left(W / 8.25 d_{2}\right)\right)$ is the current distribution coefficient between the dipole arm and the matching loop.

Putting everything together, we derive the power transmission coefficient $\tau(W, L, d)$ of the antenna as a function of the dimensions of the T-match:

$$
\tau(W, L, d)=\frac{4 \operatorname{Re}\left\{Z_{a}(W, L, d)\right\} \operatorname{Re}\left\{Z_{\text {chip }}\right\}}{\left|Z_{a}(W, L, d)+Z_{\text {chip }}\right|^{2}} .
$$

Analysis of the T-match structure indicates that the input impedance of the tag antenna can be tuned by adjusting the T-match geometry parameters, such as $\mathbf{W}, \mathbf{L}$, or line width $\mathbf{d}$. Actually, in the antenna design practice, we can use any dipole-based geometries as radiation structure and tune the impedance through T-match structure.

In this paper, Monza 5 chip (with the impedance of $Z_{\text {chip }}=14.56-161.25 j$ at the resonant frequency of 


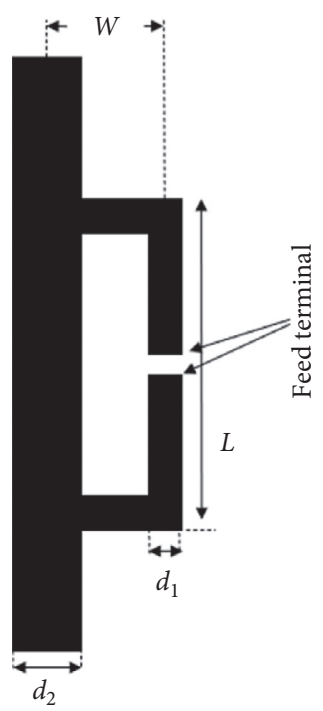

(a)

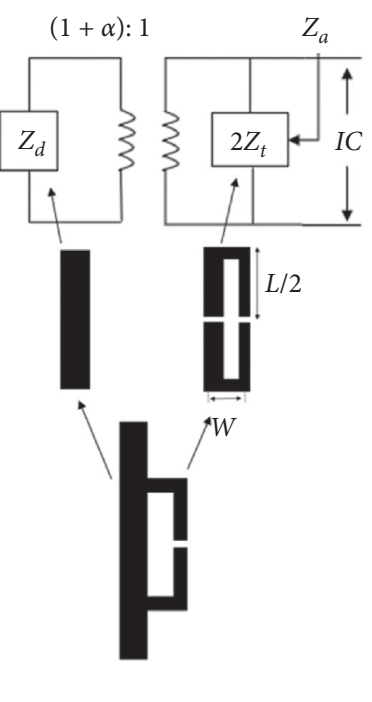

(b)

Figure 1: T-match: (a) basic structure and (b) equivalent circuit.

$915 \mathrm{MHz})$ is used as the tag chip. The antenna impedance should be optimized to the conjugate impedance of the chip, that is, $Z_{\text {chip }}=14.56+161.25 j$, to maximize the power transmission efficiency.

\section{Procedure of Letter-Shaped Antenna Design}

Antennas need to achieve certain EM properties, such as bandwidth, radiation directivity, and gains. It is unpractical and unrealistic to design antennas into arbitrary geometries and optimize all parameters in one design; compromise and trade-offs have to be considered [11].

In the rest part of this paper, we use the 26 English letters as examples and demonstrate how conforming and compromising can be achieved in designing these letter-shaped antennas.

3.1. Symmetry of Antenna Shapes. MLD antennas are naturally symmetrical, so if a letter's intrinsic shape is symmetrical, as in the case of $\mathrm{B}, \mathrm{E}, \mathrm{I}$, or T, the center of the symmetry can be naturally used as the feeding point, and a T-match structure can be constructed around the center of the symmetrical geometry; we use the T-shaped antenna as an example of symmetrical antenna designs, as shown in Figure 2(a).

For the nonsymmetrically shaped letters, their geometries can normally be separated into two parts, that is, the symmetrical part and the asymmetrical part. We use the letter $\mathrm{F}$ as an example, as shown in Figure 2(b); the geometry of $\mathrm{F}$ can be decomposed into a rotated $\mathrm{T}$ shape (shape in red in Figure 2(b) and a rectangle at the top (in black)). MLD antenna can be designed to be conformal to the T shape, as in the case of Figure 2(a). The extra rectangular shape can be treated as parasitic elements. Unavoidably, the extra asymmetrical elements will create unbalanced impedance and directivity; this imbalance can be fine-tuned, as we demonstrate in later examples of this paper.

Quite often, there are more than one way to divide a nonsymmetrical geometry into symmetrical parts and asymmetrical parts. For example, letter $\mathrm{F}$ can also be decomposed into I shape with two extra elements as shown in Figure 2(c). Designers need to evaluate these two cases and find the best selection in terms of performance and appearance.

3.2. Category of Antenna Shapes. The 26 English letters can be first divided into symmetrical and nonsymmetrical geometries:

Symmetrical-shaped letters are A, B, C, D, E, H, I, K, M, $\mathrm{O}, \mathrm{T}, \mathrm{U}, \mathrm{V}, \mathrm{W}, \mathrm{X}$, and $\mathrm{Y}$

Nonsymmetrical-shaped letters are F, G, J, L, N, P, Q, R, $\mathrm{S}$, and $\mathrm{Z}$

Normally, the largest dimension of a letter, that is, the largest separation between any two points of the letter geometry, will be used as the arm of the MLD antenna that is conformal to the letter geometry. The length of the inductive loop will be similar to a dipole antenna, that is, around halfwavelength at operating frequency $915 \mathrm{MHz}$. The width of the inductive loop can be adjusted to match the complex chip impedance in order to achieve maximum power transmission. The selection of a particular size needs trial and error to achieve the best performance.

Simulation results of different width of T-matching structure are shown in Figures 3 and 4. It can be observed that the resonant frequency shifts to the higher side with the increased width of the dipole arm in Figure 3. Meanwhile, resonant frequency shows the same relationship with the increased width of the loop arm, as shown in Figure 4.

In summary, the design practice can be summarized into the flowchart in Figure 5. 


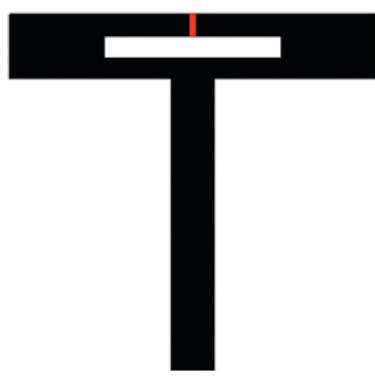

(a)

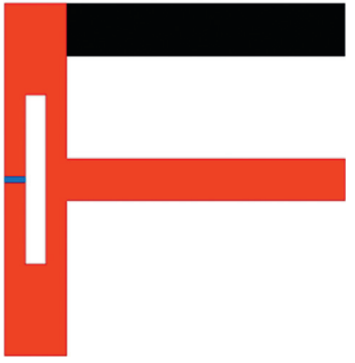

(b)

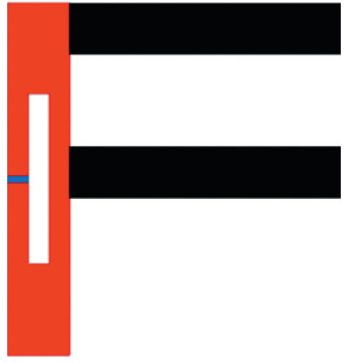

(c)

Figure 2: (a) "T" letter-shaped antenna with a feeding point and a T-match structure (symmetrical structures), (b) “T" structure, and (c) "I" structure in a nonsymmetrical structure, that is, "F."

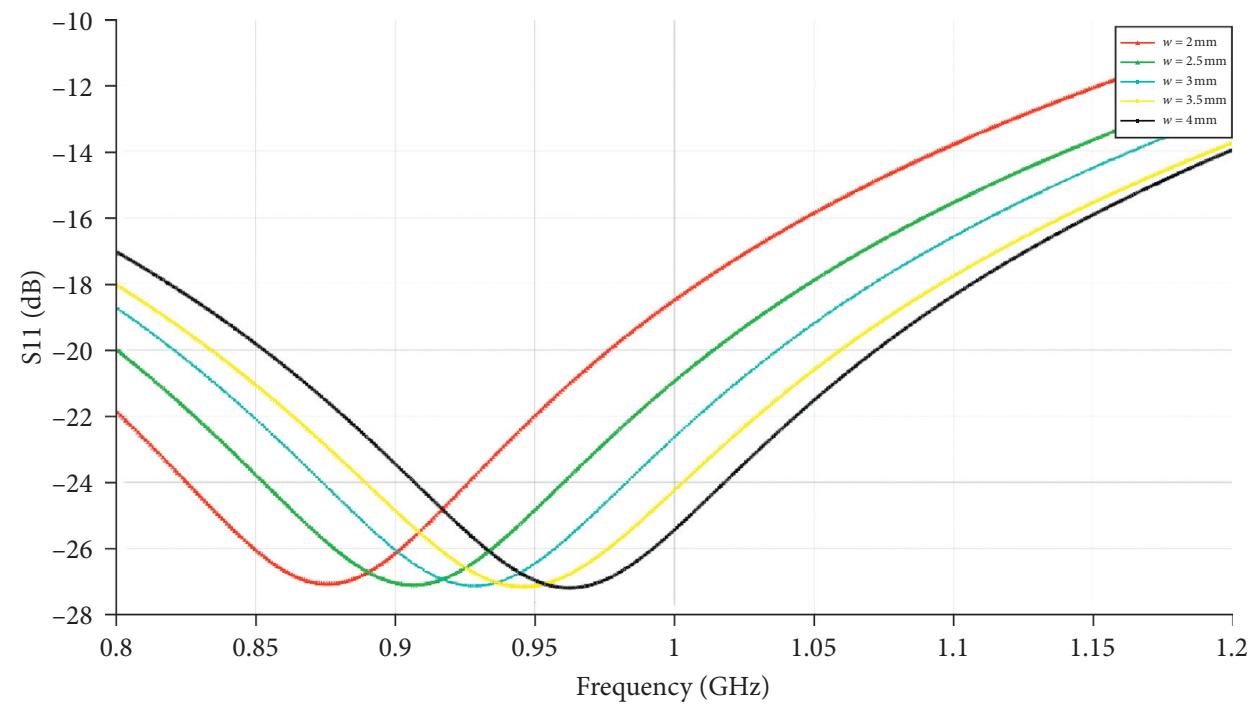

Figure 3: S11 with different dipole arm widths.

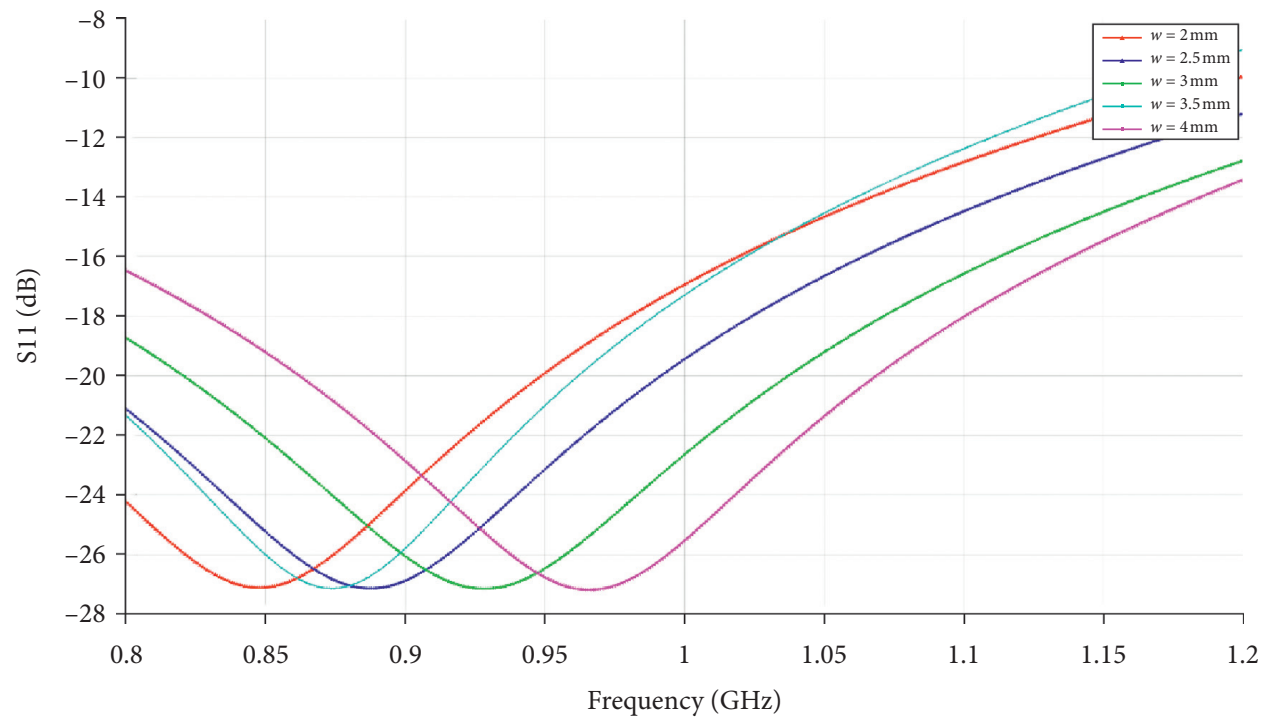

FIgURE 4: S11 with different loop widths. 


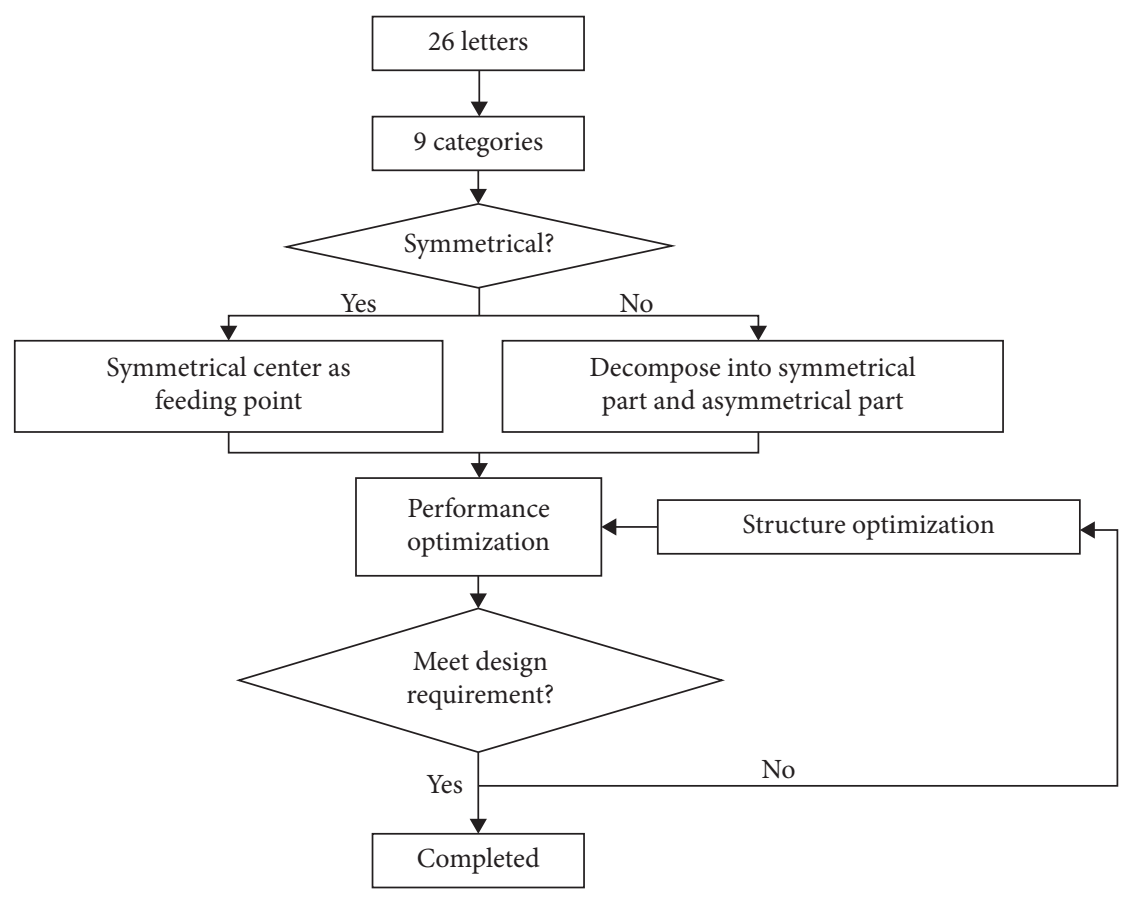

FIGURE 5: Letter-shaped tag antenna design guide.

Besides the symmetrical properties, the 26 letters can be further categorized into 9 groups, based on the similarity between these letters, as well as their corresponding geometry characteristics, as listed in Table 1.

\section{Prototyping and Performance Analysis}

4.1. Prototypes and Simulations of the 9 Templates. The 9 antenna design templates from each of the 9 groups are shown in Figure 6(a). The prototypes are fabricated through laser engraving on aluminum-laminated films, as shown in Figure 6(b). All antennas use the T-match structure for impedance matching and optimization.

Simulation results of the reflection coefficient of all 9 tag antennas are shown in Figure 7. While all antennas can achieve at least $-20 \mathrm{~dB}$ at $915 \mathrm{MHz}$, letters " $\mathrm{M}$ " and "S" have the return loss of $-36 \mathrm{~dB}$ and $-48 \mathrm{~dB}$ at $915 \mathrm{MHz}$ particularly.

Figure 8 shows the simulated $3 \mathrm{D}$ radiation pattern of all 9 letter-shaped antenna designs, where the antenna orientation is illustrated at the lower-right corner of each figure. It can be seen from the results that letters "H," "J," "M," "S," "T," and "Y" all demonstrate dipole-like radiation patterns, which are omnidirectional in horizontal plane along with two main lopes in vertical plane. Particularly, letters "E," "P," and " $U$ " exhibit irregular radiation patterns, which can be attributed to their unbalanced structures. In addition, letters "J" and " $\mathrm{M}$ " achieve the highest gain $(0.41 \mathrm{dBi}$ and $0.38 \mathrm{dBi}$, resp.), as seen in the figures. These results are in accordance with our measurement results in later sections.

4.2. Performance Discussion. Impinj Monza 5 RFID chips are mounted directly on the aluminum antennas using conductive adhesives. The tag antennas are then affixed to an FR4 substrate and are placed at a distance of $0.88 \mathrm{~m}$ from the reader. The tests are performed in an anechoic chamber, as shown in Figure 9. The interrogating frequency of the reader is swept from $860 \mathrm{MHz}$ to $960 \mathrm{MHz}$ with a $5 \mathrm{MHz}$ spacing; the bandwidth and sensitivity of each tag prototype under different frequencies are measured.

Tag's sensitivity can be derived through the threshold measurement, which is the minimum power transmitted from the reader that can power up the chip. Meanwhile, the tag reading range could also be calculated from the sensitivity using the Friis equations.

Measurement results are shown in Figures 10 and 11, where the threshold power and reading range of each lettershaped tag antenna under different frequencies are shown. In comparison, a typical commercial tag with the MLD antenna of a similar dimension, that is, "C50," is also measured and shown in the figure as a reference. The geometry of C50 antenna is also illustrated in Figure 9.

Through the threshold measurement results in Figure 10, we can observe that some letter shapes, such as " $\mathrm{M}$ " and "J," actually outperform the reference C50 antennas, that is, around 3-5 dB more sensitive at the $915 \mathrm{MHz}$ UHF RFID operation frequency. In the meanwhile, some letter shapes are hard to optimize, such as T, S, and P. Although T shape is used as the baseline geometry of many other shapes, the shape itself is not a good radiator, unless other peripheral geometries are added.

The reading range of each letter-shaped tag antenna is illustrated in Figure 11. Most of the antennas reach the maximum reading range around $915 \mathrm{MHz}$. More specifically, " $\mathrm{M}$ " and "J" achieve the longest reading range to more than 20 meters. Other letter-shaped tag antennas have various reading ranges from 3 meters to 10 meters, 
TABLE 1: Letter geometry can be categorized into 9 groups.

\begin{tabular}{lc}
\hline Categories & Characteristic \\
\hline U CVG & Structure of concaved shapes \\
P ODQ & Structure with a single loop \\
E BF & Structures with "T" shapes embedded \\
M KW & Symmetrical structures with bended arms \\
S ZN & Line structure with two turns \\
H X & Structures with four arms, symmetrical both vertically and horizontally \\
J L & Line structure with one turn \\
Y A & Symmetrical in one dimension \\
T I & T and I structure can be used as the baseline structure of other variations \\
\hline
\end{tabular}

Note: we chose one letter shape from each of the 9 groups as the antenna template, as marked in bold text.

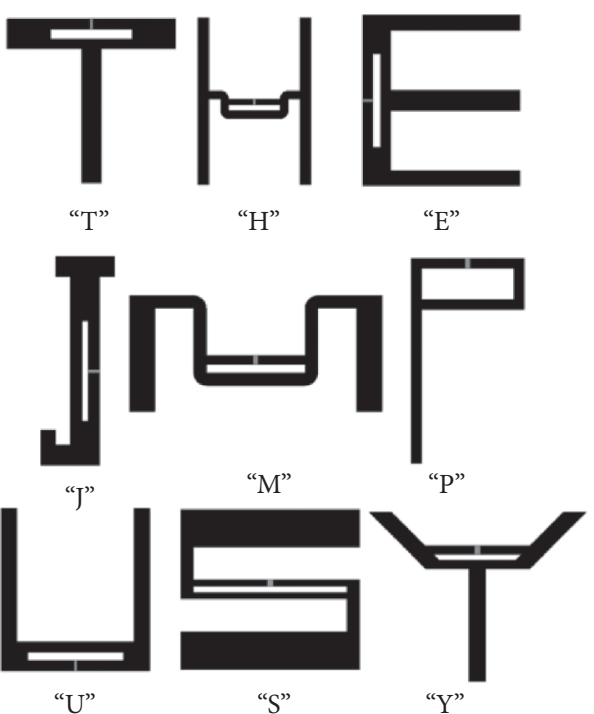

(a)

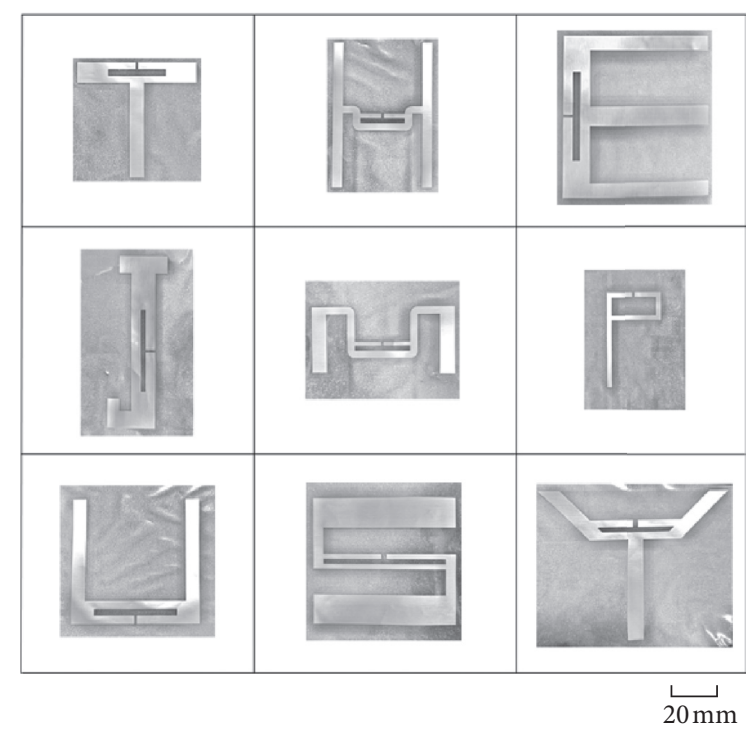

(b)

FIgURE 6: (a) Nine template antennas from each of the 9 groups. (b) Nine tag antenna prototypes.

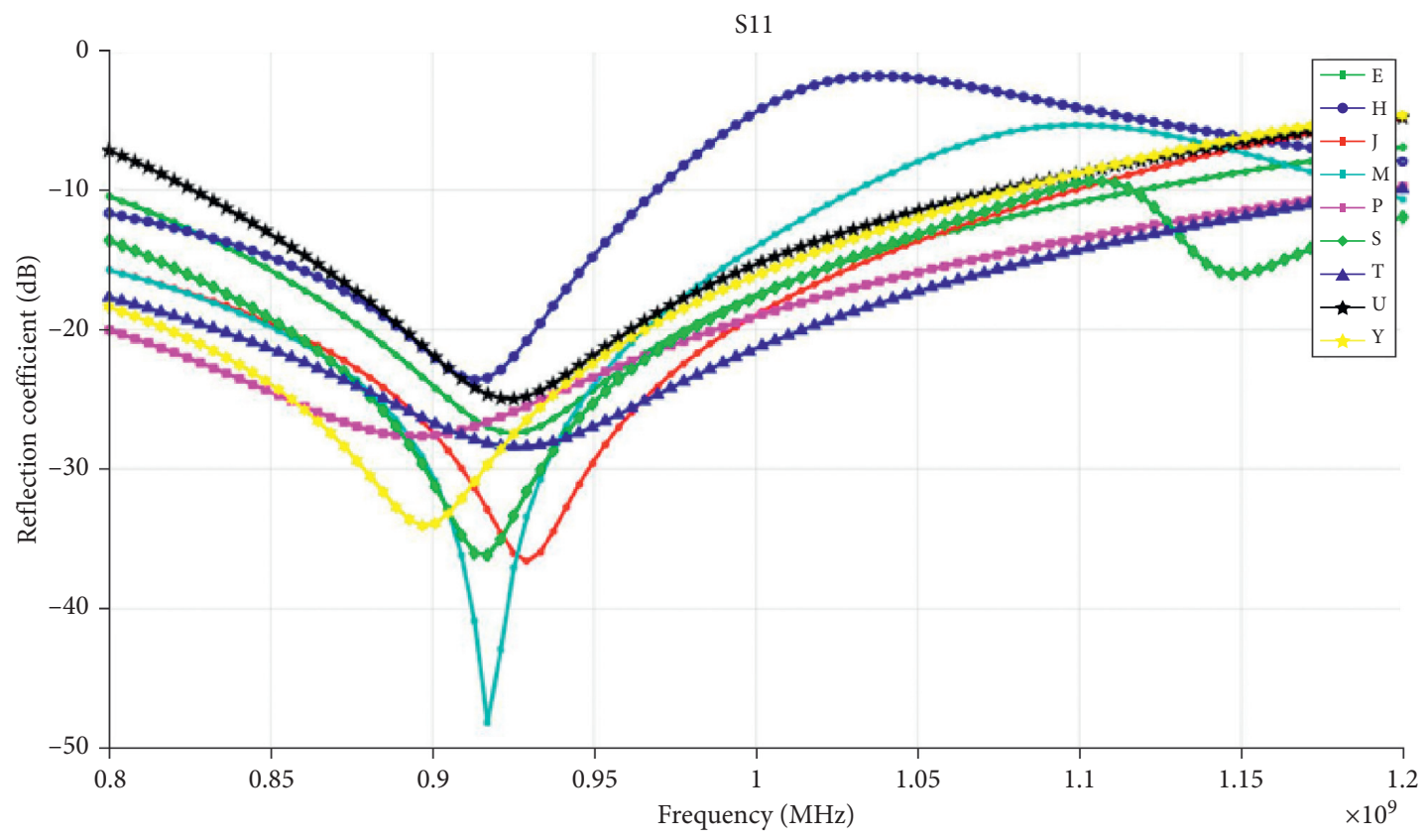

FIGURE 7: Simulated reflection coefficient of 9 template tag antennas. 


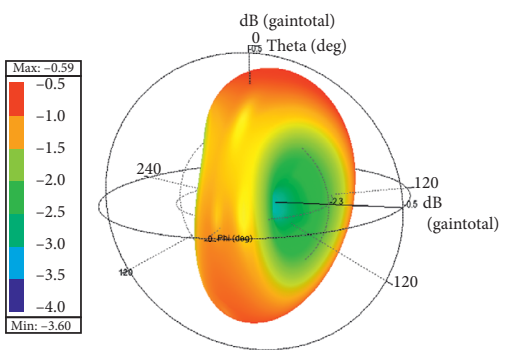

(a)

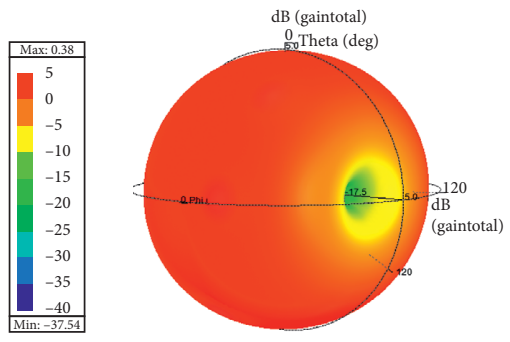

(d)

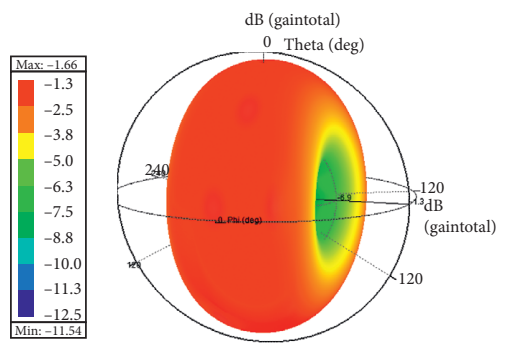

(g)

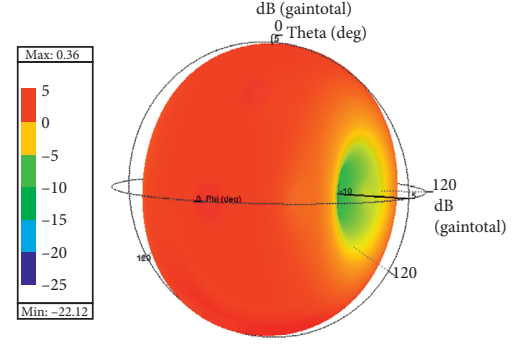

(b)
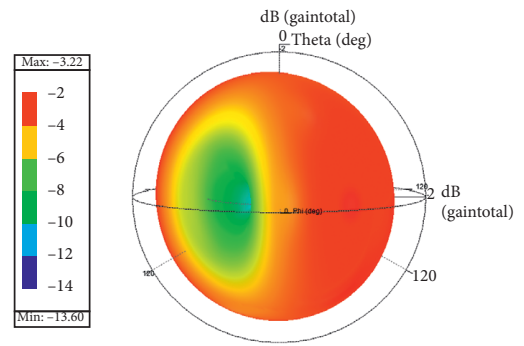

(e)
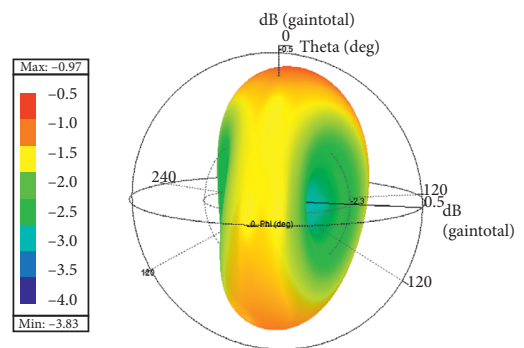

(h)

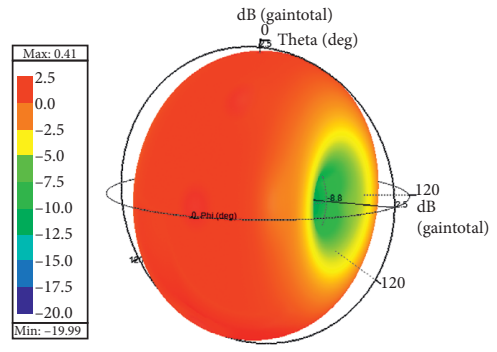

(c)
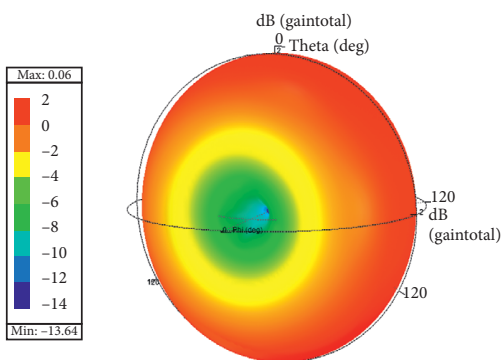

(f)

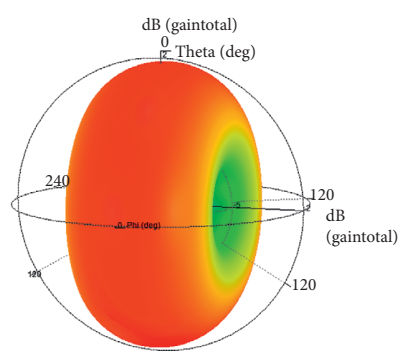

(i)

FIGURE 8: Simulated radiation patterns of 9 letter-shaped antennas. (a) E gain plot. (b) H gain plot. (c) J gain plot. (d) M gain plot. (e) P gain plot. (f) S gain plot. (g) T gain plot. (h) U gain plot. (i) Y gain plot.

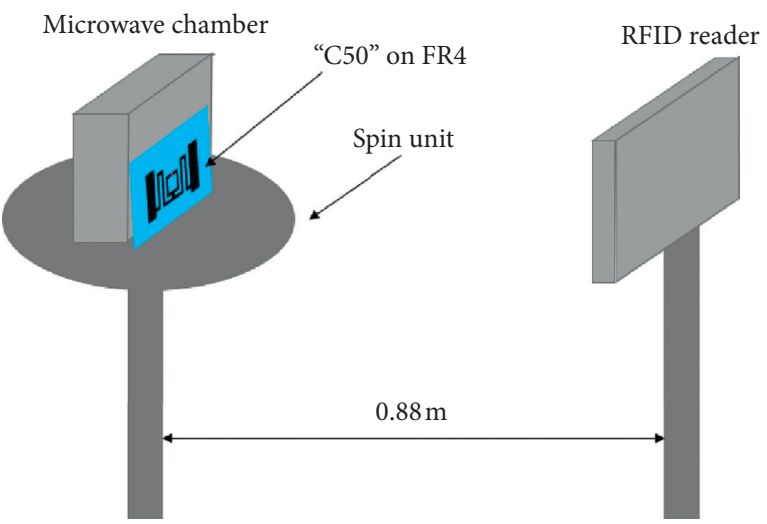

(a)

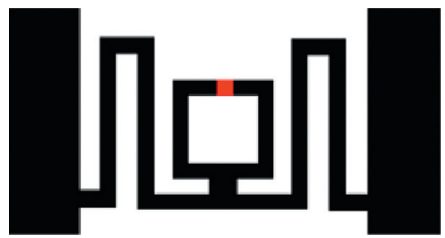

"C50"

(b)

FIgURE 9: Measurement setup and C50 layout. 


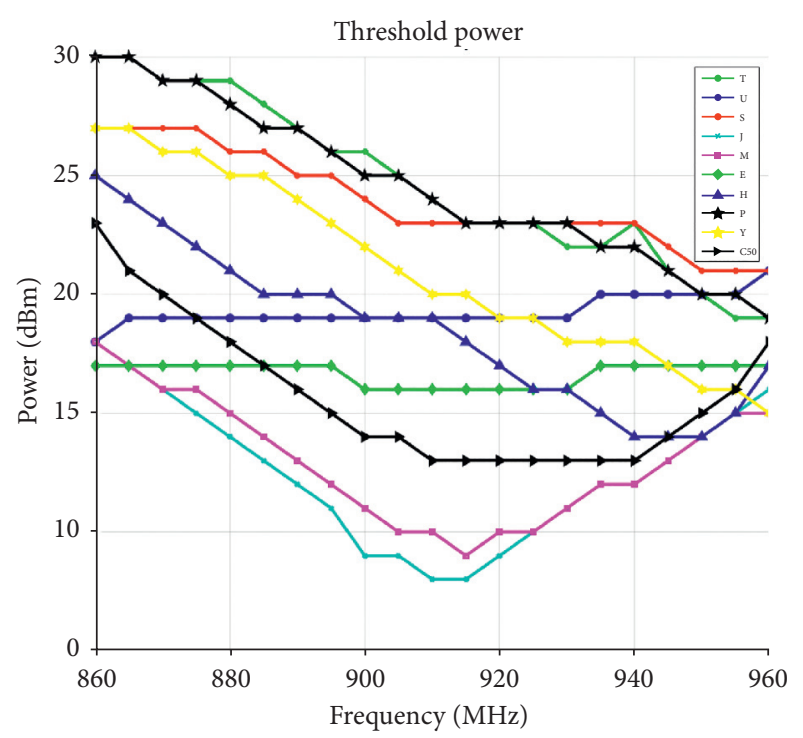

Figure 10: Threshold power of tag antennas.

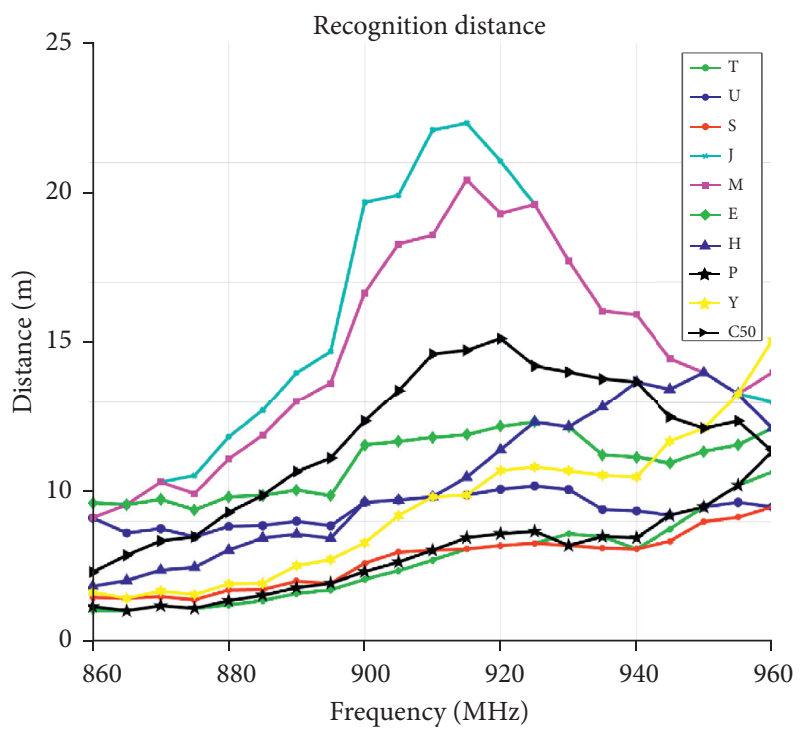

Figure 11: Read range of tag antennas.

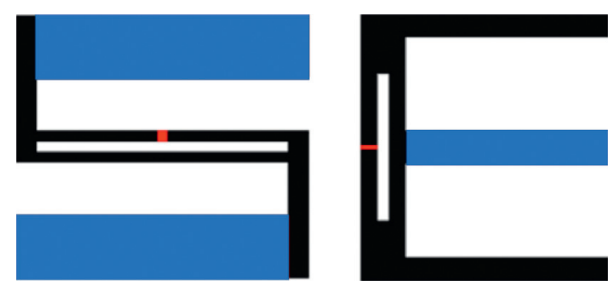

FIgURE 12: Inductive structures in "S" and "E."

respectively. The differences in the antenna performance can be attributed to different letter geometries; that is, letter "J" shape performs like a standard dipole antenna, while " $\mathrm{S}$ " structure is composed of a dipole antenna and two bended radiation arms. These two bended arms are couple with the inductive loop and their radiation will interfere with each other and consequently deteriorate the performance $[16,17]$, as shown in Figure 12.

\section{Conclusion}

In this work, we propose the design methodology of UHF RFID tag antennas with unconventional geometries. We use the shapes of the 26 English letters as examples to demonstrate how the performance of the antenna and conformance of geometry can be achieved and balanced. We categorized the 26 letter shapes into 9 groups and design antenna prototypes from each of the 9 groups. The performances of these letter-shaped antennas are comparable or sometimes even better than the standard MLD antennas. This methodology can also be extended to other unconventionally shaped antenna designs.

\section{Data Availability}

The antenna data used to support the findings of this study can be obtained from the author via email (12032213@ mail.sustech.edu.cn).

\section{Conflicts of Interest}

The authors declare that they have no conflicts of interest.

\section{Acknowledgments}

This work was supported in part by the Key-Area Research and Development Program of Guangdong Province (Grant No. 2020B0101030002); in part by the Shenzhen Science and Technology Program (Grant No. JCYJ20190809115803580); and in part by the High-Level University Fund under Grant G02236002.

\section{References}

[1] H. Rmili, B. Oussama, J. Yousaf et al., "Robust detection for chipless RFID tags based on compact printable Alphabets," Sensors, vol. 19, no. 21, p. 4785, 2019.

[2] K. Koski, A. Vena, L. Sydänheimo, L. Ukkonen, and Y. Rahmat-Samii, "Design and implementation of electrotextile ground planes for wearable UHF RFID patch tag antennas," IEEE Antennas and Wireless Propagation Letters, vol. 12, pp. 964-967, 2013.

[3] F. Paredes, G. Zamora, F. J. Herraiz-Martinez, F. Martin, and J. Bonache, "Dual-band UHF-RFID tags based on meanderline antennas loaded with spiral resonators," IEEE Antennas and Wireless Propagation Letters, vol. 10, pp. 768-771, 2011.

[4] J. Xi and H. Zhu, "UHF RFID impedance matching: T-matchdipole tag design on the highway," in Proceedings of the 2015 IEEE International Conference on RFID (RFID), pp. 86-93, San Diego, CA, USA, April 2015.

[5] C. Calabrese and G. Marrocco, "Meandered-slot antennas for sensor-RFID tags," IEEE Antennas and Wireless Propagation Letters, vol. 7, pp. 5-8, 2008.

[6] Y. Liu, M. Yu, L. Xu et al., "Characterizations and optimization techniques of embroidered RFID antenna for wearable applications," IEEE Journal of Radio Frequency Identification, vol. 4, no. 1, pp. 38-45, 2019. 
[7] J. Xi and T. T. Ye, "Conformal UHF RFID tag antenna mountable on winebottle neck," in Proceedings of the 2012 IEEE International Symposium on Antennas and Propagation, pp. 1-2, Chicago, IL, USA, July 2012.

[8] K. L. Chung and C.-H. Wong, "Wang-shaped patch antenna for wireless communications," IEEE Antennas and Wireless Propagation Letters, vol. 9, pp. 638-640, 2010.

[9] F. Mutlu, M. A. Demir, and Ö. Ergül, "Improved fonts for chipless radio-frequency-identification tags based on letters," in Proceedings of the 2018 18th Mediterranean Microwave Symposium (MMS), pp. 247-250, Istanbul, Turkey, October 2018.

[10] S. Tedjini, O. Boularess, T. Andriamiharivolamena, H. Rmili, and T. Aguili, "A novel design of chipless RFID tags based on alphabets," in Proceedings of the 2017 IEEE MTT-S International Microwave Symposium (IMS), pp. 1561-1563, Honololu, HI, USA, June 2017.

[11] M. Keskilammi and M. Kivikoski, "Using text as a meander line for RFID transponder antennas," IEEE Antennas and Wireless Propagation Letters, vol. 3, pp. 372-374, 2004.

[12] T. Singh, S. Tedjini, E. Perret, and A. Vena, "A frequency signature based method for the RF identification of letters," in Proceedings of the 2011 IEEE International Conference on RFID, pp. 1-5, Orlando, FL, USA, April 2011.

[13] K. L. Chung, W. Li, Y. Li, R. Liu, and P. Zhang, "Chinese character-shaped artistic patch antenna," IEEE Antennas and Wireless Propagation Letters, vol. 18, no. 8, pp. 1542-1546, 2019.

[14] K. V. S. Rao, P. V. Nikitin, and S. F. Lam, "Antenna design for UHF RFID tags: a review and a practical application," IEEE Transactions on Antennas and Propagation, vol. 53, no. 12, pp. 3870-3876, 2005.

[15] C. A. Balanis, Antenna Theory: Analysis and Design, John Wiley \& Sons, Hoboken, NJ, USA, 2016.

[16] H.-W. Son and C.-S. Pyo, "Design of RFID tag antennas using an inductively coupled feed," Electronics Letters, vol. 41, no. 18, pp. 994-996, 2005.

[17] G. Marrocco, "The art of UHF RFID antenna design: impedance-matching and size-reduction techniques," IEEE Antennas and Propagation Magazine, vol. 50, no. 1, pp. 66-79, 2008. 